\title{
Laparoscopic Uterosacral Ligament Suspension versus Sacrospinous Ligament Fixation for Apical Prolapse: Perioperative Outcomes
}

\section{Martina Gabra ( $\sim$ mgabra@arizona.edu )}

University of Arizona Medical Center - University Campus: Banner University Medical Center Tuscon https://orcid.org/0000-0002-8940-6976

\section{Veronica Winget}

University of Arizona Medical Center - University Campus: Banner University Medical Center Tuscon

\section{Mohammad T Torabi}

The University of Arizona

\section{llana Addis}

University of Arizona Medical Center - University Campus: Banner University Medical Center Tuscon

Kenneth Hatch

University of Arizona Medical Center - University Campus: Banner University Medical Center Tuscon John Heusinkveld

University of Arizona Medical Center - University Campus: Banner University Medical Center Tuscon

\section{Original Article}

Keywords: Laparoscopic uterosacral ligament suspension, perioperative complications, sacrospinous ligament fixation

Posted Date: February 18th, 2021

DOl: https://doi.org/10.21203/rs.3.rs-208585/v1

License: (a) (1) This work is licensed under a Creative Commons Attribution 4.0 International License. Read Full License 


\section{Abstract}

Background: Pelvic organ prolapse can be repaired vaginally or laparoscopically. Studies comparing vaginal repair with sacrospinous ligament fixation (SSLF) or uterosacral ligament suspension (V-USLS) have found no difference in functional or adverse outcomes. Laparoscopic USLS (L-USLS) is becoming a popular treatment for pelvic organ prolapse because it has a low rate of ureteral compromise. To date, no studies have compared perioperative outcomes between L-USLS and SSLF. The objective of this study is to compare the rates of perioperative complications (intraoperative and postoperative complications) between these two methods.

Methods: This was a retrospective chart review of 243 consecutive patients who underwent L-USLS or SSLF at one institution between 3/2017 and 8/2019 for apical pelvic organ prolapse. Descriptive data was analyzed as appropriate with student t-tests and Chi Square. Univariable Logistic Regression analysis was performed to assess predictors of perioperative complications.

Results: Preoperative Pelvic Organ Prolapse Quantification Stage (POP-Q) was similar between the two cohorts $(p=0.23)$. After adjusting for confounding factors, L-USLS was associated with a longer operative time (118 vs $142 \mathrm{~min}, p<0.01)$ and shorter length of hospitalization ( 0.68 vs 1.06 days, $p<0.01)$. The estimated blood loss between the procedures was not statistically significant after adjusting for confounding factors. There was no difference in perioperative complication rates between L-USLS and $\operatorname{SSLF}(5 \%$ vs $7 \%, p=0.55)$. No clinical risk factors were significantly associated with perioperative complications.

Conclusion: We did not find a difference in complications between L-USLS and SSLF.

\section{Background}

Pelvic organ prolapse can affect quality of life by leading to urinary retention, constipation, discomfort with intercourse, or vaginal erosion and infection. Up to $13 \%$ of women will undergo surgery for pelvic organ prolapse in their lifetime. ${ }^{1}$ Surgical repairs are performed with native tissue or synthetic mesh. Due to concerns regarding mesh complications, more patients are choosing to avoid the use of synthetic mesh for pelvic organ prolapse repair. ${ }^{2}$ Vaginal uterosacral ligament suspension (V-USLS) and sacrospinous ligament fixation (SSLF) are methods of native tissue treatment of apical pelvic organ prolapse. Barber et al found no difference in anatomic, functional, or adverse event outcomes at 2 year follow up between V-USLS and SSLF. ${ }^{3}$ The laparoscopic approach to USLS (L-USLS) is gaining more widespread use because it allows full visualization of the uterosacral ligament, allowing suspension at a higher point along the uterosacral ligament. Studies comparing L-USLS to V-USLS showed a lower risk of ureteral compromise compared to V-USLS. ${ }^{4-6}$ In addition, Turner et al found no significant difference in prolapse recurrence between L-USLS and V-USLS. ${ }^{7}$ To date, there are no studies comparing perioperative complications and surgical outcomes between L-USLS and SSLF to guide patient counseling. The 
primary objective of this study was to determine the rate of perioperative complications between L-USLS and SSLF.

\section{Methods}

A retrospective chart review was performed on 243 consecutive patients who underwent L-USLS or SSLF from 3/1/2017 to 8/31/2019. All surgeries were performed at Banner-University Medical Center Tucson, an academic hospital system associated with the University of Arizona. There are three faculty that perform the majority of urogynecologic procedures $(\mathrm{JH}, \mathrm{KH}, \mathrm{IA})$. University of Arizona and Banner Hospital Institutional Review Board exemption was granted for this study.

All patients were evaluated in clinic by an attending physician preoperatively. They were assessed for Pelvic Organ Prolapse Quantification (POP-Q) Stage. ${ }^{9}$ Patients were evaluated for occult stress urinary incontinence at the discretion of their physician. Leading edge information was translated to POP-Q Stage. Demographic, surgical and medical history, intraoperative course, postoperative complications, and postoperative follow up data was collected from the electronic medical record. Pertinent medical history was defined as any diagnoses from the Charlson Comorbidity Index because it has been shown to be an independent predictor of surgical mortality as well as long-term survival. ${ }^{8}$ These diagnoses include: history of myocardial infarction, congestive heart failure, peripheral vascular disease, transient ischemic attack, dementia, chronic obstructive pulmonary disease, connective tissue disease, peptic ulcer disease, liver disease, diabetes mellitus, chronic kidney disease, hemiplegia, or current cancer.

L-USLS was performed bilaterally, except in one case where unilateral suspension was performed due to adhesions. L-USLS was performed by plicating the bilateral uterosacral ligaments with 2-0 Polyethylene terephthalate suture. SSLF was performed bilaterally in all but ten cases and involved suspending the vaginal apex to the sacrospinous ligaments with the Capio suture-capturing device and 0-polydioxanone suture. Resident physicians participated in all surgical cases. The majority of patients underwent concomitant procedures including: posterior colporrhaphy, anterior colporrhaphy, midurethral sling, or salpingo-oophorectomy.

The primary objective of this study was to evaluate the overall rate of perioperative complications. Overall perioperative complication rate was assessed to allow better detection of differences between the two surgical approaches. Perioperative complications include both intraoperative and postoperative complications within 30 days of surgery. Complications were defined as: conversion to laparotomy, cystotomy, ureteral injury/kinking/need for suture release, bowel injury, take back to the operating room, blood transfusion, deep vein thrombosis (DVT), pulmonary emboli (PE), ileus or small bowel obstruction (SBO), wound infection, hernia formation, pelvic abscess, and hospital re-admission. Other outcome data that was collected included: post-operative buttock pain, pelvic pain or dyspareunia, urinary tract infection, and urinary retention. Urinary tract infection was defined as patient report of urinary symptoms and a positive urine culture. Urinary retention was defined as inability to spontaneously void postoperatively requiring discharge to home with a catheter. 
$P$-values were calculated from student t-tests for continuous variables and Chi Square for categorical variables. Univariable Logistic Regression analysis was performed to assess predictors of perioperative complications. A $p$ value $<0.05$ was considered statistically significant. Statistical analysis was performed using SPSS Statistical software, v.27.0 Armonk, NY, IBM Inc

\section{Results}

Between 3/2017-8/2019, 243 patients underwent L-USLS or SSLF. One patient in the L-USLS cohort was excluded due to planned concomitant bowel resection and 10 patients in the SSLF cohort were excluded (7 due to vaginal mesh use, 1 due to vaginal mesh excision, 1 due to concomitant umbilical hernia repair, and 1 due to sphincteroplasty). A total of 113 women in the L-USLS and 109 women in the SSLF cohort were included in the analysis. Overall, baseline characteristics were similar between the two groups (Table 1). Women in the SSLF cohort were older than those in the L-USLS cohort (63 vs 59, $p=0.02$ ). In addition, women who underwent SSLF were more likely to be post-menopausal, have a prior hysterectomy, and have a prior surgery for pelvic organ prolapse (all with $p<0.05$ ). There were no differences in POPQ measurements between groups, $p=0.86$.

Concomitant procedures and intraoperative data are presented in Table 2. Hysterectomy was performed in $87 \%$ of patients in the L-USLS cohort and $54 \%$ of patients in the SSLF cohort $(p<0.01)$. Uterine sparing hysteropexy was performed in 9 patients; 2 in the L-USLS group and 7 in the SSLF group. Fewer anterior and posterior vaginal repairs were required for L-USLS compared to SSLF ( $4 \%$ vs $61 \%$ and $45 \%$ vs $71 \%$ respectively, both $p<0.01$ ). Total operative time was longer for the L-USLS cohort (140 vs 118 minutes, $p$ $<0.01$ ). In addition, average blood loss was lower in the L-USLS cohort (120 vs $152 \mathrm{~mL}, p=0.02$ ) and length of admission was shorter in the L-USLS cohort ( 0.68 vs 1.06 days, $p=<0.01$ ). After adjusted for age, co-morbidities, and concurrent procedures (hysterectomy, anterior repair, posterior repair, BSO, and MUS), the length of admission and operative time remained statistically significant, but blood loss was no longer statistically significant.

There was a similar rate of perioperative complications in both groups ( $5 \% \mathrm{vs} 7 \%, p=0.55$ ), Table 3 . Using the Clavien-Dindo grading scale, in the L-USLS cohort there were two Grade 1 complications, five Grade 2 complications and two Grade 3 complication. In the SSLF cohort, there were two Grade 1 complications four Grade 2 complication, and three Grade 3 complications. There was one cystotomy in both the LUSLS and SSLF cohorts, and one bowel injury in the SSLF cohort. All were recognized intraoperatively and repaired without postoperative sequelae. One patient in the SSLF cohort was taken back to the OR due bleeding at the vaginal cuff that was identified in the post-operative care unit. One patient in the LUSLS group experienced a small bowel obstruction which required bowel resection. This patient had a complex surgical history with resulting dense abdominal adhesions. There were two readmissions in each group; two for pneumonia, one for enterocolitis, and one for vaginal bleeding which did not require any intervention. Univariable logistic regression comparing outcomes between the two groups did not identify risk factors for complications (data not shown). Multivariable logistic regression was not performed due to the small number of complications identified. 
Postoperative urinary retention was common; it affected $24 \%$ of women in the L-USLS cohort and $15 \%$ of women in the SSLF cohort $(p=0.08)$. Two women in the L-USLS cohort experienced persistent urinary retention after sling placement and required sling release. Urinary retention resolved in all women in the SSLF cohort. Urinary tract infection occurred in 5\% of women in the L-USLS cohort and $9 \%$ of women in the SSLF cohort $(p=0.27)$. Nine women in the SSLF cohort experienced gluteal pain post-operatively, one required removal of sutures due to persistent pain. Two women in each cohort experienced new pelvic pain post-operatively, one woman in the L-USLS cohort required local analgesic injection for treatment.

The median follow up in this cohort was 7 weeks (IQR 5.4-22.2 weeks), defined as date of surgery to last office appointment. Immediate post-operative POP-Q information was available for $89 \%$ of patients in the L-USLS cohort and $91 \%$ of patients in the SSLF cohort. At last clinic follow up, approximately $82 \%$ of patients in both groups had Stage 0 or Stage 1 prolapse $(p=0.76)$. In patients with follow up beyond 3 months, we aimed to characterize the short-term rate of recurrent prolapse. Prolapse recurrence was defined as prolapse of any compartment (anterior, apical, or posterior) beyond the hymen or retreatment for prolapse by either surgery or pessary. Recurrent pelvic organ prolapse occurred in 8 patients in the LUSLS cohort and 9 patients in the SSLF cohort (7\% vs 7\%, $p=0.43$ ). Apical prolapse occurred in 5 patients in the L-USLS cohort, four underwent laparoscopic sacrocolpopexy and one underwent SSLF. Two patients in the SSLF cohort had apical prolapse, one underwent repeat SSLF and one elected for conservative management with physical therapy.

\section{Discussion}

There was no statistically significant difference in perioperative complications between L-USLS and SSLF in this study. After adjusting for confounding variables, there was no statistically significant difference in blood loss between the cohorts, but L-USLS remained associated with a longer operative time and shorter length of hospital admission. The length of hospital admission in the SSLF group was likely confounded by physician practice to admit patients who have undergone extensive vaginal repairs or vaginal hysterectomy for overnight observation.

It is notable that patients in the SSLF cohort were more likely to undergo a concomitant anterior or posterior colporrhaphy to achieve appropriate surgical repair. However, the SSLF cohort had similar shortterm prolapse recurrence rate compared to L-USLS on follow up. These results should be interpreted with caution due to limited post-operative follow up data and the non-standardized post-operative examination reporting. In addition all L-USLS and SSLF procedures were included, regardless of whether a concomitant hysterectomy or hysteropexy was performed, which may alter the recurrence rate.

Operative time for L-USLS in this study was similar to that reported by Barber et al for V-USLS (140 min vs 146 min, respectively). ${ }^{3}$ The length of hospital admission in the L-USLS group was shorter than that reported for V-USLS (0.7 vs 2.4 days). This shortened length of admission was notable because $87 \%$ of patients underwent hysterectomy, however $38 \%$ were discharged on post-operative day 0 and $60 \%$ were discharged on post-operative day 1 . In addition, there was no ureteral injury or compromise in the L-USLS 
cohort, which is consistent with prior studies that report a $0 \%$ rate of ureteral compromise. ${ }^{6,10-12}$ The low rate of ureteral injury is postulated to be due to the laparoscopic approach allowing visualization of the ureter course to avoid ureter injury or kinking. This is a potential advantage over V-USLS as it has been associated with a $3-8 \%$ incidence of ureteral compromise. ${ }^{3,13}$

Strengths of this study include well defined intraoperative and postoperative complications. Complications would be identified intra-operatively or during the inpatient post-operative period. This study is limited by its retrospective nature; data collected was limited to that documented in the chart. In addition there were some patients with limited long-term clinical follow up. Patients were referred for treatment by their primary gynecologists and resumed care with the referring physician after they were judged to be fully recovered from surgery. It is therefore possible that some postoperative complications or recurrences were treated at an outside facility. Finally, there is a potential for selection bias- the factors that led a surgeon and patient to choose L-USLS or SSLF were not formally assessed.

\section{Conclusion}

In conclusion, there was not a statistically significant difference in perioperative complications between L-USLS and SSLF A post-hoc power analysis was performed and 2,200 subjects per arm would be needed to detect a difference in perioperative complications with $80 \%$ power and alpha 0.05 . Given these unattainable sample sizes, the outcomes of this study are likely clinically similar. Randomized controlled trials comparing L-USLS and SSLF are needed to determine long-term prolapse recurrence rates.

\section{List Of Abbreviations}

L-USLS: Laparoscopic uterosacral ligament suspension

V-USLS: vaginal uterosacral ligament suspension

SSLF: Sacrospinous ligament fixation

POP-Q: Pelvic Organ Prolapse Quantification Stage

BMl: Body mass index

DVT: Deep vein thrombosis

PE: Pulmonary emboli

SBO: small bowel obstruction

EBL: estimated bloos loss

MUS: Midurethral sling 
BSO: Bilateral salpingo-oophorectomy

\section{Declarations}

1. Wu JM, Matthews CA, Conover MM, Pate V, Jonsson Funk M. Lifetime risk of stress urinary incontinence or pelvic organ prolapse surgery. Obstet Gynecol. Jun 2014;123(6):1201-6. doi:10.1097/AOG.0000000000000286

2. Skoczylas LC, Turner LC, Wang L, Winger DG, Shepherd JP. Changes in prolapse surgery trends relative to FDA notifications regarding vaginal mesh. Int Urogynecol J. Apr 2014;25(4):471-7. doi:10.1007/s00192-013-2231-7

3. Barber MD, Brubaker L, Burgio KL, et al. Comparison of 2 transvaginal surgical approaches and perioperative behavioral therapy for apical vaginal prolapse: the OPTIMAL randomized trial. JAMA. Mar 2014;311(10):1023-34. doi:10.1001/jama.2014.1719

4. Sze EH, Karram MM. Transvaginal repair of vault prolapse: a review. Obstet Gynecol. Mar 1997;89(3):466-75. doi:10.1016/S0029-7844(96)00337-7

5. Diwan A, Rardin CR, Strohsnitter WC, Weld A, Rosenblatt P, Kohli N. Laparoscopic uterosacral ligament uterine suspension compared with vaginal hysterectomy with vaginal vault suspension for uterovaginal prolapse. Int Urogynecol J Pelvic Floor Dysfunct. Jan 2006;17(1):79-83. doi:10.1007/s00192-005-1346-x

6. Barbier HM, Smith MZ, Eto CU, et al. Ureteral Compromise in Laparoscopic Versus Vaginal Uterosacral Ligament Suspension: A Retrospective Cohort. Female Pelvic Med Reconstr Surg. 2015 Nov-Dec 2015;21(6):363-8. doi:10.1097/SPV.0000000000000202

7. Turner LC, Lavelle ES, Shepherd JP. Comparison of complications and prolapse recurrence between laparoscopic and vaginal uterosacral ligament suspension for the treatment of vaginal prolapse. Int Urogynecol J. May 2016;27(5):797-803. doi:10.1007/s00192-015-2897-0

8. Charlson ME, Pompei P, Ales KL, MacKenzie CR. A new method of classifying prognostic comorbidity in longitudinal studies: development and validation. J Chronic Dis. 1987;40(5):373-83. doi:10.1016/0021-9681(87)90171-8

9. Bump RC, Mattiasson A, Bø K, et al. The standardization of terminology of female pelvic organ prolapse and pelvic floor dysfunction. Am J Obstet Gynecol. Jul 1996;175(1):10-7. doi:10.1016/s0002-9378(96)70243-0

10. Rardin CR, Erekson EA, Sung VW, Ward RM, Myers DL. Uterosacral colpopexy at the time of vaginal hysterectomy: comparison of laparoscopic and vaginal approaches. J Reprod Med. May 2009;54(5):273-80.

11. Filmar GA, Fisher HW, Aranda E, Lotze PM. Laparoscopic uterosacral ligament suspension and sacral colpopexy: results and complications. Int Urogynecol J. Dec 2014;25(12):1645-53. doi:10.1007/s00192-014-2407-9 
12. Lin LL, Phelps JY, Liu CY. Laparoscopic vaginal vault suspension using uterosacral ligaments: a review of 133 cases. J Minim Invasive Gynecol. 2005 May-Jun 2005;12(3):216-20.

doi:10.1016/j.jmig.2005.03.014

13. Barber MD, Visco AG, Weidner AC, Amundsen CL, Bump RC. Bilateral uterosacral ligament vaginal vault suspension with site-specific endopelvic fascia defect repair for treatment of pelvic organ prolapse. Am J Obstet Gynecol. Dec 2000;183(6):1402-10; discussion 1410-1. doi:10.1067/mob.2000.111298

\section{Tables}

Due to technical limitations, full-text HTML conversion of the tables could not be completed. However, the table can be downloaded and accessed in the Supplementary Files.

\section{Supplementary Files}

This is a list of supplementary files associated with this preprint. Click to download.

- Table1.png

- Table2.png

- Table3.png

- Table4.png 